\title{
Hexadecyldimethyl benzyl ammonium bromide: an efficient catalystfor a clean one-pot synthesis of tetrahydrobenzopyran derivatives in water
}

\author{
Tong-Shou Jin*, Ai-Qing Wang, Feng Shi, Li-Sha Han, Li-Bin Liu, and Tong-Shuang Li \\ College of Chemistry and Environmental Science, Hebei University, Baoding 071002, P. R. \\ China \\ E-mail: jintongshou@yahoo.com.cn
}

\begin{abstract}
A clean and efficient method for the synthesis of 2-amino-4-aryl-3-cyano-5-oxo- 4H-5,6,7,8tetrahydrobenzopyran derivatives using hexadecyldimethylbenzyl ammonium bromide (HDMBAB) as the catalyst is described. This method provides several advantages, such as simple work-up procedure, environmentally benign, neutral conditions and high yields. In addition, water was chosen as a green solvent.
\end{abstract}

Keywords: Tetrahydrobenzopyran, aromatic aldehyde, malononitrile, 1,3-cyclohexanedione, 5,5-dimethyl-1,3-cyclohexanedione; aqueous media

\section{Introduction}

In recent years, $4 H$-benzopyran and its derivatives have attracted strong interest due to their useful biological and pharmacological properties, such as anticoagulant, spasmolytic, diuretic, anticancer, antianaphylactin agents. ${ }^{1}$ Some 2 -amino- $4 H$-pyrans can be employed as photoactive materials. ${ }^{2}$ Furthermore, substituted $4 \mathrm{H}$-pyrans also constitute a structural unit of a series of natural products. ${ }^{3,4}$ The synthesis of $4 H$-benzopyrans has been reported; ${ }^{5}$ the conventional methods are with base as catalyst (piperidine or triethylamine) in an organic solvent such as ethanol, acetic acid or DMF, ${ }^{6-8}$ and using ultrasonic irradiation. ${ }^{9}$ Each of the above methods has its own merit, but some are not entirely satisfactory, owing to such drawbacks as low yields, difficult work-up, problems of corrosiveness and effluent pollution. Consequently, there is a need to develop alternative methods for the synthesis of tetrahydrobenzopyran derivatives under mild and environmentally friendly conditions.

Water has been applied to organic reactions as a solvent, and it has several advantages such as its low cost, safety, non-polluting nature and operational simplicity. ${ }^{10}$ In 1983, Breslow discovered that the Diels-Alder reaction performed in water was subject to a huge rate 
acceleration. ${ }^{11}$ This observation led to increased interest from synthetic organic chemists in organic reactions conducted in water. Soon it was discovered that other organic reactions, like the Claisen rearrangement, ${ }^{12}$ the aldol condensation, ${ }^{13}$ Diels-Alder reaction, ${ }^{14}$ the benzoin condensation, ${ }^{15}$ Mannich reaction ${ }^{16}$ and Michael reaction ${ }^{17}$ exhibit rate enhancements in water. To date, many more organic reactions have been carried out in water. ${ }^{18}$

In this manuscript, we wish to report a general and highly efficient route for the synthesis of tetrahydrobenzopyrans using an inexpensive, commercially available hexadecyldimethylbenzyl ammonium bromide (HDMBAB) catalyst. This is a one-pot reaction in aqueous media, which is not only operationally simple but also consistently gives the corresponding products in good to excellent yields (Scheme 1).

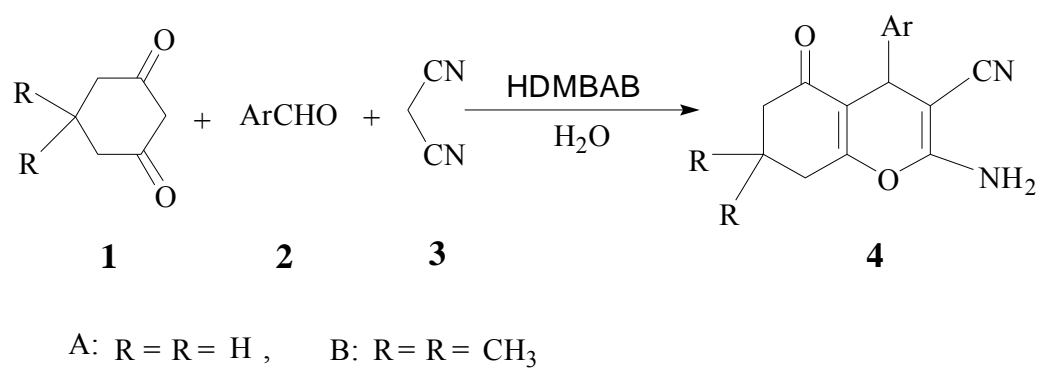

\section{Scheme 1}

In a typical general experimental procedure, an aqueous solution of 1,3-cyclohexanedione or 5,5-dimethyl-1,3-cyclohexanedione 1, aromatic aldehyde 2 and malononitrile 3 was heated at $80-90{ }^{\circ} \mathrm{C}$ in the presence of a catalytic amount of HDMBAB. The corresponding 2-amino-4-aryl3-cyano-5-oxo-4H-5,6,7,8-tetrahydrobenzopyran derivatives 4 were obtained in good to excellent yields and the results are summarized in Table 1.

As shown in Table 1, the three-component cyclocondensation reaction proceeded smoothly at $80-90{ }^{\circ} \mathrm{C}$ in water to give the corresponding products 4 in high yields. The electronic nature of the substituents on the aromatic ring did not show a strong effect in terms of yields under these reaction conditions. Both aromatic aldehydes containing electron-withdrawing groups (such as nitro group, halide) or electron-donating groups (such as alkyl group, alkoxy group) gave good to excellent yields of the corresponding $4 H$-tetrahydrobenzopyrans.

The catalyst plays a crucial role in the success of the reaction in terms of the rate and the yields. Taking the reaction of 4-nitrobenzaldehyde with 1,3-cyclohexanedione 2 and $\mathbf{3}$ as an example, the reaction could be carried out in the absence of the catalyst when the mixture was heated in water for $6 \mathrm{~h}$, but a very poor yield resulted (18\%). We have tested some catalysts such as tetrabutyl ammonium bromide, benzyltrimethyl ammonium chloride, sodium dodecyl sulfate and hexadecyldimethylbenzyl ammonium bromide, and the yields using these four catalysts were $78 \%, 86 \%, 65 \%$ and $96 \%$, respectively. From the yield data, it was found that 
hexadecyldimethylbenzyl ammonium bromide (HDMBAB) was the best catalyst for this reaction.

Table1. Synthesis of 4H-tetrahydrobenzopyrans catalyzed by HDMBAB in water

\begin{tabular}{|c|c|c|c|c|c|c|c|}
\hline \multirow[t]{2}{*}{ Entry } & \multirow[t]{2}{*}{$\mathrm{Ar}$} & \multirow[t]{2}{*}{$\mathrm{R}$} & \multirow{2}{*}{$\begin{array}{l}\text { Time } \\
\text { (h) }\end{array}$} & \multirow[t]{2}{*}{ Product } & \multirow{2}{*}{$\begin{array}{c}\text { Yield }^{\mathrm{a}} \\
(\%)\end{array}$} & \multicolumn{2}{|c|}{$\mathrm{Mp}\left({ }^{\circ} \mathrm{C}\right)$} \\
\hline & & & & & & Found & Reported $^{19}$ \\
\hline 1 & $2-\mathrm{ClC}_{6} \mathrm{H}_{4} \mathbf{1 a}$ & $\mathrm{H}$ & 6.0 & $4 a$ & 92 & $210-212$ & $213-215$ \\
\hline 2 & $4-\mathrm{ClC}_{6} \mathrm{H}_{4} \mathbf{1 b}$ & $\mathrm{H}$ & 6.0 & $4 b$ & 94 & $224-226$ & $226-229$ \\
\hline 3 & $2,4-\mathrm{Cl}_{2} \mathrm{C}_{6} \mathrm{H}_{3} \mathbf{1 c}$ & $\mathrm{H}$ & 6.0 & $4 c$ & 95 & $223-225$ & $225-227$ \\
\hline 4 & $3-\mathrm{NO}_{2} \mathrm{C}_{6} \mathrm{H}_{4} \mathbf{1 d}$ & $\mathrm{H}$ & 5.5 & 4d & 96 & $202-204$ & $198-200$ \\
\hline 5 & $4-\mathrm{NO}_{2} \mathrm{C}_{6} \mathrm{H}_{4} \mathbf{1 e}$ & $\mathrm{H}$ & 5.5 & $4 e$ & 96 & $234-236$ & $234-235$ \\
\hline 6 & $4-\mathrm{CH}_{3} \mathrm{OC}_{6} \mathrm{H}_{4} \mathbf{1 f}$ & $\mathrm{H}$ & 7.0 & $4 f$ & 88 & $192-194$ & $193-195$ \\
\hline 7 & $3,4-\mathrm{OCH}_{2} \mathrm{OC}_{6} \mathrm{H}_{3} \mathbf{1 g}$ & $\mathrm{H}$ & 7.0 & $4 g$ & 86 & $210-212$ & $211-214$ \\
\hline 8 & $2-\mathrm{ClC}_{6} \mathrm{H}_{4} \mathbf{1 h}$ & $\mathrm{CH}_{3}$ & 7.5 & $4 h$ & 88 & $216-218$ & $215-216$ \\
\hline 9 & $3-\mathrm{ClC}_{6} \mathrm{H}_{4} \mathbf{1 i}$ & $\mathrm{CH}_{3}$ & 7.5 & $4 \mathbf{i}$ & 89 & $222-224$ & $224-225$ \\
\hline 10 & $4-\mathrm{ClC}_{6} \mathrm{H}_{4} \mathbf{1} \mathbf{j}$ & $\mathrm{CH}_{3}$ & 7.5 & $4 j$ & 90 & $237-239$ & $239-241$ \\
\hline 11 & $2-\mathrm{NO}_{2} \mathrm{C}_{6} \mathrm{H}_{4} \mathbf{1} \mathbf{k}$ & $\mathrm{CH}_{3}$ & 7.0 & $4 \mathbf{k}$ & 90 & $182-184$ & $180-182$ \\
\hline 12 & $3-\mathrm{NO}_{2} \mathrm{C}_{6} \mathrm{H}_{4} \mathbf{1 l}$ & $\mathrm{CH}_{3}$ & 7.0 & 41 & 92 & $212-214$ & $213-214$ \\
\hline 13 & $4-\mathrm{NO}_{2} \mathrm{C}_{6} \mathrm{H}_{4} \mathbf{1 m}$ & $\mathrm{CH}_{3}$ & 7.0 & $4 m$ & 93 & $178-180$ & $176-178$ \\
\hline 14 & $4-\mathrm{CH}_{3} \mathrm{OC}_{6} \mathrm{H}_{4} \mathbf{1 n}$ & $\mathrm{CH}_{3}$ & 8.0 & $4 n$ & 86 & 194-196 & 196-198 \\
\hline 15 & $4-\mathrm{CH}_{3} \mathrm{C}_{6} \mathrm{H}_{4} 1 \mathbf{0}$ & $\mathrm{CH}_{3}$ & 8.0 & 40 & 84 & $218-220$ & $220-222$ \\
\hline 16 & $3,4-\mathrm{OCH}_{2} \mathrm{OC}_{6} \mathrm{H}_{3} \mathbf{1 p}$ & $\mathrm{CH}_{3}$ & 8.0 & $4 p$ & 85 & $220-222$ & $221-223$ \\
\hline
\end{tabular}

a Isolated yield. 
We have studied also the effect of the amount of the catalyst on these reactions. With the mixture 1e, 1,3-cyclohexanedione and 3 in the presence of $3 \mathrm{~mol} \%$ HDMBAB, the product $4 \mathbf{e}$ was obtained in $77 \%$ yield at $80-90{ }^{\circ} \mathrm{C}$ in water for $5.5 \mathrm{~h}$. Increasing the amount of catalyst to $5 \mathrm{~mol} \%, 8 \mathrm{~mol} \%$ and $12 \mathrm{~mol} \%$, yields of $84 \%, 88 \%$ and $96 \%$, respectively, were obtained. Use of just $12 \mathrm{~mol} \%$ HDMBAB is sufficient to push the reaction forward and higher amounts of the catalyst did not improve the results to any greater extent. Thus, $12 \mathrm{~mol} \%$ HDMBAB was chosen as the amount of catalyst for these reactions.

We propose the possible following mechanism to account for the reaction. First, the aromatic aldehyde 2 is condensed with malononitrile (3) to afford the $\alpha$-cyanocinnamonitrile derivative 5 . The step $(\mathbf{2}+\mathbf{3} \rightarrow \mathbf{5})$ can be regarded as a rapid Knoevenagel reaction. Since, in a model reaction, the Knoevenagel reaction of malononitrile and aromatic aldehydes can be carried out in water without any catalyst, we conjecture that the second step requires the presence of $\mathrm{HDMBAB}$. The active methylene of 1 reacts with the electrophilic $\mathrm{C}=\mathrm{C}$ double bond in $\mathbf{5}$ giving the intermediate 6, which tautomerizes into 7. The latter is then cyclized by nucleophilic attack of the $\mathrm{OH}$ group on the cyano $(\mathrm{CN})$ moiety, giving intermediate 8. Finally, the expected product 4 is afforded by tautomerization $(\mathbf{8} \rightarrow \mathbf{4})$. HDMBAB not only is a phase transfer catalyst, but it serves also as an emulsifier in this reaction process (Scheme 2).
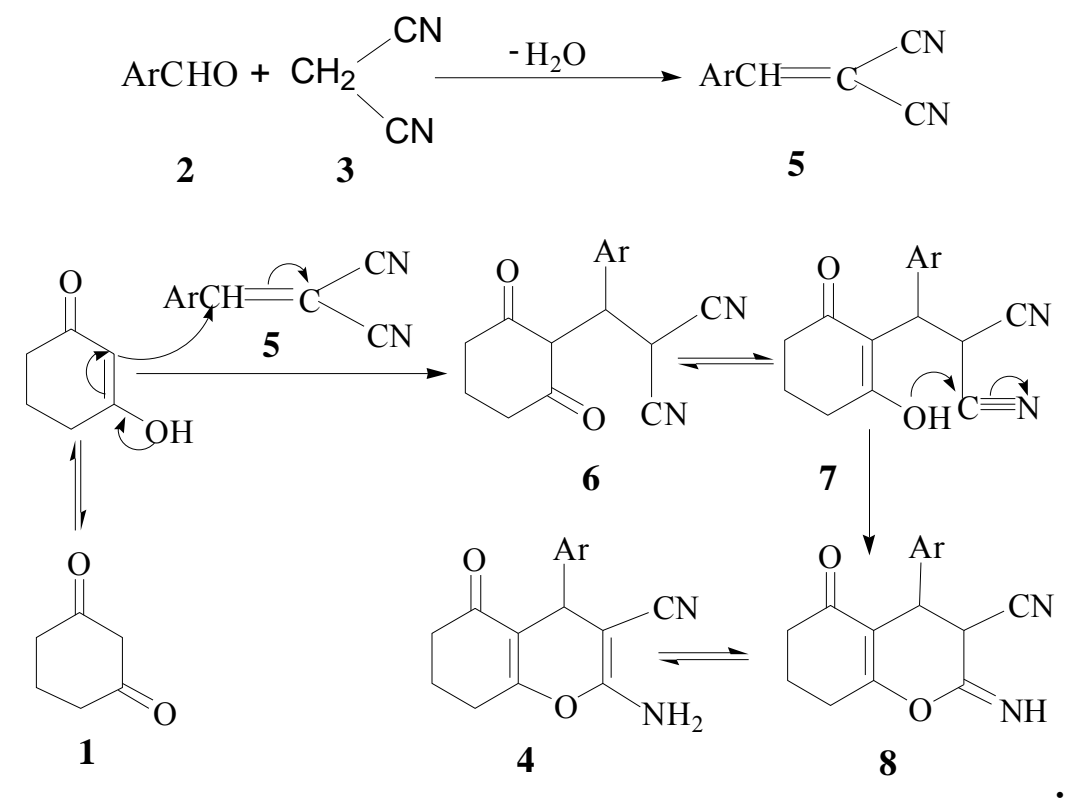

\section{Scheme 2}

In conclusion, we have described a procedure for the preparation of $4 H$-benzopyran derivatives catalyzed by HDMBAB, using a three-component condensation in heated water. In addition, it is possible to apply the tenets of green chemistry to the generation of biologically interesting products using aqueous media approaches that are less expensive and less toxic than 
those with organic solvents. Moreover, the procedure offers several advantages including high yields, operational simplicity, clean reaction conditions and minimum pollution of the environment, which makes it a useful and attractive process for the synthesis of these compounds.

\section{Experimental Section}

General Procedures. IR spectra were recorded on a Bio-Rad FTS-40 spectrometer (KBr). ${ }^{1} \mathrm{H}$ NMR spectra were measured on a Bruker AVANCE 400 (400 MHz) spectrometer using TMS as internal reference and DMSO- $d_{6}$ as solvent. Elemental analyses were determined using PerkinElmer 2400 II elemental analyzer.

\section{General procedure for synthesis of tetrahydrobenzopyran derivatives}

A mixture of 5,5-dimethyl-1,3-cyclohexadione or 1,3-cyclohexadione (1,5 mmol), aromatic aldehyde (2, $5 \mathrm{mmol})$, malononitrile (3, $5 \mathrm{mmol})$, and HDMBAB (12 mol\%) in water (30 $\mathrm{mL})$ was stirred at $80-90{ }^{\circ} \mathrm{C}$ for $5.5-8.0 \mathrm{~h}$. Then the mixture was cooled to room temperature, solid was filtered off and washed with $\mathrm{H}_{2} \mathrm{O}(40 \mathrm{ml})$. The crude products were purified by recrystallization from ethanol $(95 \%)$ to afford pure products 4 . Data for all compounds are shown below:

2-Amino-4-(2-chlorophenyl)-3-cyano-5-oxo-4H-5,6,7,8-tetrahydrobenzopyran (4a). IR $(\mathrm{KBr}): v_{\max }=3312,3200,2200,1696,1600,1512,1360,1210,1000,750 \mathrm{~cm}^{-1} .{ }^{1} \mathrm{H} \mathrm{NMR}$ (DMSO- $\left.d_{6}\right): \delta_{\mathrm{H}}=1.88-2.02(\mathrm{~m}, 2 \mathrm{H}, \mathrm{CH}-7), 2.16-2.34$ (m, 2H, CH-8), 2.50-2.68 (m, 2H, CH-6), 4.72 (s, 1H, CH-4), 7.00 (s, 2H, NH ), 7.18-7.28 (m, 3H, ArH), 7.34-7.36 (m, 1H, ArH) ppm. Anal. calcd. for $\mathrm{C}_{16} \mathrm{H}_{13} \mathrm{ClN}_{2} \mathrm{O}_{2}$ : C 63.90, $\mathrm{H}$ 4.36, N 9.31; found $\mathrm{C} 64.02, \mathrm{H} \mathrm{4.32,} \mathrm{N} \mathrm{9.37} \mathrm{\% .}$

2-Amino-4-(4-chlorophenyl)-3-cyano-5-oxo-4H-5,6,7,8-tetrahydrobenzopyran (4b). IR $(\mathrm{KBr}): v_{\max }=3416,3340,2200,1684,1606,1600,1495,1360,1260,1206,1000,912,780,750$ $\mathrm{cm}^{-1} .{ }^{1} \mathrm{H}$ NMR (DMSO-d $\left.)_{6}\right) \delta_{\mathrm{H}}=1.88-2.00(\mathrm{~m}, 2 \mathrm{H}, \mathrm{CH}-7), 2.22-2.34$ (m, 2H, CH-8), 2.60-2.72 (m, 2H, CH-6), 4.22 (s, 1H, CH-4), 7.02 (s, 2H, NH $)$, 7.18-7.20 (m 2H, ArH), 7.32-7.36 (m, 2H, ArH) ppm. Anal. calcd. for $\mathrm{C}_{16} \mathrm{H}_{13} \mathrm{ClN}_{2} \mathrm{O}_{2}$ : C 63.90, $\mathrm{H} 4.36, \mathrm{~N} 9.31$; found $\mathrm{C} 64.00, \mathrm{H} \mathrm{4.38,} \mathrm{N}$ $9.27 \%$.

2-Amino-4-(2,4-dichlorophenyl)-3-cyano-5-oxo-4H-5,6,7,8-tetrahydrobenzopyran (4c). IR $(\mathrm{KBr}): v_{\max }=3350,3200,2212,1706,1650,1610,1508,1468,1416,1280,1004,810,760 \mathrm{~cm}^{-}$ 1. ${ }^{1} \mathrm{H}$ NMR (DMSO- $\left.d_{6}\right): \delta_{\mathrm{H}}=1.94-2.00(\mathrm{~m}, 2 \mathrm{H}, \mathrm{CH}-7), 2.24-2.34(\mathrm{~m}, 2 \mathrm{H}, \mathrm{CH}-8), 2.56-2.66(\mathrm{~m}$, 2H, CH-6), 4.70 (s, 1H, CH-4), 7.06 (s, 2H, NH ), 7.22-7.24 (m, 1H, ArH), 7.34-7.36 (m, 1H, ArH), 7.50-7.52 (m, 1H, ArH). Anal. calcd. for $\mathrm{C}_{16} \mathrm{H}_{12} \mathrm{Cl}_{2} \mathrm{~N}_{2} \mathrm{O}_{2}$ : C 57.33, $\mathrm{H}$ 3.61, N 8.36; found C 57.42,H 3.66, N $8.43 \%$.

2-Amino-3-cyano-4-(3-nitrophenyl)-5-oxo-4H-5,6,7,8-tetrahydrobenzopyran (4d). IR (KBr): $v_{\max }=3360,3308,2200,2190,1686,1640,1510,1450,1402,1370,1260,1140,850,780,750$ $\mathrm{cm}^{-1} .{ }^{1} \mathrm{H}$ NMR (DMSO-d $\left.)_{6}\right) \delta_{\mathrm{H}}=1.88-2.01$ (m, 2H, CH-7), 2.20-2.35 (m, 2H, CH-8), 2.60-2.70 
(m, 2H, CH-6), 4.52 (s, 1H, CH-4), 7.15 (s, 2H, NH $), 7.60-7.68$ (m, 2H, ArH), 7.98 (s, 1H, ArH), 8.06-8.08 (m, 1H, ArH) ppm. Anal. calcd. for $\mathrm{C}_{16} \mathrm{H}_{13} \mathrm{~N}_{3} \mathrm{O}_{4}: \mathrm{C} 61.73, \mathrm{H} 4.21, \mathrm{~N} 13.50$; found C 61.78, H 4.16, N $13.57 \%$.

2-Amino-3-cyano-4-(4-nitrophenyl)-5-oxo-4H-5,6,7,8-tetrahydrobenzopyran (4e). IR ( $\mathrm{KBr}$ ): $v_{\max }=3400,3330,2200,1702,1690,1590,1510,1436,1350,1210,1000,820,790,740 \mathrm{~cm}^{-1}$. ${ }^{1} \mathrm{H}$ NMR (DMSO-d $\left.d_{6}\right): \delta_{\mathrm{H}}=1.94-1.98(\mathrm{~m}, 2 \mathrm{H}, \mathrm{CH}-7), 2.28-2.32(\mathrm{~m}, 2 \mathrm{H}, \mathrm{CH}-8), 2.62-2.66(\mathrm{~m}$, $2 \mathrm{H}, \mathrm{CH}-6), 4.38$ (s, 1H, CH-4), 7.12 (s, 2H, NH 2$), 7.44$ (d, 2H, J=8.0Hz, ArH), 8.14 (d, 2H, $J=8.0 \mathrm{~Hz}$, ArH) ppm. Anal. calcd. for $\mathrm{C}_{16} \mathrm{H}_{13} \mathrm{~N}_{3} \mathrm{O}_{4}$ : C 61.73, H 4.21, N 13.50; found C 61.81, $\mathrm{H}$ 4.26, N $13.57 \%$.

2-Amino-3-cyano-4-(4-methoxyphenyl)-5-oxo-4H-5,6,7,8-tetrahydrobenzopyran (4f). IR $(\mathrm{KBr}): v_{\max }=3460,3320,2220,1700,1600,1510,1450,1368,1245,1026,840,770,750 \mathrm{~cm}^{-1}$. ${ }^{1} \mathrm{H}$ NMR (DMSO-d $): \delta_{\mathrm{H}}=1.93-1.96(\mathrm{~m}, 2 \mathrm{H}, \mathrm{CH}-7), 2.26-2.30(\mathrm{~m}, 2 \mathrm{H}, \mathrm{CH}-8), 2.58-2.62(\mathrm{~m}$, $2 \mathrm{H}, \mathrm{CH}-6), 3.74\left(\mathrm{~s}, 3 \mathrm{H}, \mathrm{OCH}_{3}\right), 4.20(\mathrm{~s}, 1 \mathrm{H}, \mathrm{CH}-4), 6.82(\mathrm{~d}, 2 \mathrm{H}, \mathrm{J}=8.0 \mathrm{~Hz}, \mathrm{ArH}), 6.98(\mathrm{~s}, 2 \mathrm{H}$, $\left.\mathrm{NH}_{2}\right), 7.06(\mathrm{~d}, 2 \mathrm{H}, J=8.0 \mathrm{~Hz}, \mathrm{ArH}) \mathrm{ppm}$. Anal. calcd. for $\mathrm{C}_{17} \mathrm{H}_{16} \mathrm{~N}_{2} \mathrm{O}_{3}$ : C 68.91, H 5.44, N 9.45; found C 69.00, H 5.47, N $9.49 \%$.

2-Amino-3-cyano-4-(3,4-methylenedioxyphenyl)-5-oxo-4H-5,6,7,8-tetrahydrobenzopyran (4g). IR (KBr): $v_{\max }=3360,3196,2962,2195,1650,1605,1490,1378,1360,1250,1210,1124$, 1036, 850, $780 \mathrm{~cm}^{-1} .{ }^{1} \mathrm{H}$ NMR (DMSO-d $): \delta_{\mathrm{H}}=0.98\left(\mathrm{~s}, 3 \mathrm{H}, \mathrm{CH}_{3}\right), 1.02\left(\mathrm{~s}, 3 \mathrm{H}, \mathrm{CH}_{3}\right), 2.12(\mathrm{~d}$, $1 \mathrm{H}, J=16.0 \mathrm{~Hz}, \mathrm{CH}-8), 2.22$ (d, 1H, $J=16.0 \mathrm{~Hz}, \mathrm{CH}-8), 2.46-2.56$ (m, 2H, CH -6), 4.15(s, 1H, CH4), $5.98\left(\mathrm{~s}, 2 \mathrm{H}, \mathrm{OCH}_{2} \mathrm{O}\right), 6.63(\mathrm{~d}, 1 \mathrm{H}, J=8.0 \mathrm{~Hz}, \mathrm{ArH}), 6.66(\mathrm{~s}, 1 \mathrm{H}, \mathrm{ArH}), 6.82(\mathrm{~d}, 1 \mathrm{H}, J=8.0 \mathrm{~Hz}$, ArH), 6.98 (s, 2H, $\mathrm{NH}_{2}$ ) ppm. Anal. calcd. for $\mathrm{C}_{17} \mathrm{H}_{14} \mathrm{~N}_{2} \mathrm{O}_{4}$ : C 65.80, H 4.55, N 9.03; found C 65.87, H 4.46, N $9.11 \%$.

2-Amino-4-(2-chlorophenyl)-3-cyano-7,7-dimethyl-5-oxo-4H-5,6,7,8-tetrahydrobenzopyran (4h). IR (KBr): $v_{\max }=3330,3186,2962,2200,1660,1560 \mathrm{~cm}^{-1} .{ }^{1} \mathrm{H}$ NMR (DMSO-d $)$ : $\delta_{\mathrm{H}}=0.98\left(\mathrm{~s}, 3 \mathrm{H}, \mathrm{CH}_{3}\right), 1.02\left(\mathrm{~s}, 3 \mathrm{H}, \mathrm{CH}_{3}\right), 2.18(\mathrm{~d}, 1 \mathrm{H}, J=16.0 \mathrm{~Hz}, \mathrm{CH}-8) 2.24(\mathrm{~d}, 1 \mathrm{H}, J=16.0 \mathrm{~Hz}$, CH-8), 2.46-2.56 (m, 2H, CH-6), 4.50 (s, 1H, CH-4), 7.02 (s, 2H, NH 2$), 7.16-7.36$ (m, 4H, ArH) ppm. Anal. calcd. for $\mathrm{C}_{18} \mathrm{H}_{17} \mathrm{ClN}_{2} \mathrm{O}_{2}$ : C 65.75, $\mathrm{H} \mathrm{5.21,} \mathrm{N} \mathrm{8.52;} \mathrm{found} \mathrm{C} \mathrm{65.71,} \mathrm{H} \mathrm{5.32,} \mathrm{N} \mathrm{8.58} \mathrm{\% .}$

2-Amino-4-(3-chlorophenyl)-3-cyano-7,7-dimethyl-5-oxo-4H-5,6,7,8-tetrahydrobenzopyran (4i). IR (KBr): $v_{\max }=3390,3290,3180,2190,1660,1584 \mathrm{~cm}^{-1} .{ }^{1} \mathrm{H}$ NMR (DMSO-d $)$ ): $\delta_{\mathrm{H}}=1.08\left(\mathrm{~s}, 3 \mathrm{H}, \mathrm{CH}_{3}\right), 1.12\left(\mathrm{~s}, 3 \mathrm{H}, \mathrm{CH}_{3}\right), 2.26(\mathrm{~s}, 2 \mathrm{H}, \mathrm{CH}-8), 2.46-2.48(\mathrm{~m}, 2 \mathrm{H}, \mathrm{CH}-6), 4.43(\mathrm{~s}$, $1 \mathrm{H}, \mathrm{CH}-4), 6.58\left(\mathrm{~s}, 2 \mathrm{H}, \mathrm{NH}_{2}\right), 7.18-7.28(\mathrm{~m}, 4 \mathrm{H}, \mathrm{ArH}) \mathrm{ppm}$. Anal. calcd. for $\mathrm{C}_{18} \mathrm{H}_{17} \mathrm{ClN}_{2} \mathrm{O}_{2}$ : C 65.75, H 5.21, N 8.52; found C 65.78, H 5.31, N 8.49\%.

2-Amino-4-(4-chlorophenyl)-3-cyano-7,7-dimethyl-5-oxo-4H-5,6,7,8-tetrahydrobenzopy-

ran (4j). IR (KBr): $v_{\max }=3408,3310,3006,2200,1694,1606 \mathrm{~cm}^{-1} .{ }^{1} \mathrm{H}$ NMR (DMSO-d $\left.d_{6}\right)$ : $\delta_{\mathrm{H}}=0.97\left(\mathrm{~s}, 3 \mathrm{H}, \mathrm{CH}_{3}\right), 1.05\left(\mathrm{~s}, 3 \mathrm{H}, \mathrm{CH}_{3}\right), 2.12(\mathrm{~d}, 1 \mathrm{H}, J=16.0 \mathrm{~Hz}, \mathrm{CH}-8) 2.22(\mathrm{~d}, 1 \mathrm{H}, \mathrm{J}=16.0 \mathrm{~Hz}$, CH-8), 2.48-2.54 (m, 2H, CH-6), 4.28 (s, 1H, CH-4), 7.04 (s, 2H, NH$), 7.16$ (d, 2H, J=8.0Hz, ArH), 7.34 (d, 2H, J=8.0Hz, ArH) ppm. Anal. calcd. for $\mathrm{C}_{18} \mathrm{H}_{17} \mathrm{ClN}_{2} \mathrm{O}_{2}$ : C 65.75, H 5.21, N 8.52; found C 65.79, H 5.28, N $8.47 \%$.

2-Amino-3-cyano-7,7-dimethyl-4-(2-nitrophenyl)-5-oxo-4H-5,6,7,8-tetrahydrobenzopy- 
ran (4k). IR (KBr): $v_{\max }=3440,3310,3006,2200,1698,1604 \mathrm{~cm}^{-1} .{ }^{1} \mathrm{H}$ NMR (DMSO-d $)$ : $\delta_{\mathrm{H}}=0.96\left(\mathrm{~s}, 3 \mathrm{H}, \mathrm{CH}_{3}\right), 1.06\left(\mathrm{~s}, 3 \mathrm{H}, \mathrm{CH}_{3}\right), 2.14(\mathrm{~m}, 2 \mathrm{H}, \mathrm{CH}-8), 2.47(\mathrm{~s}, 2 \mathrm{H}, \mathrm{CH}-6), 5.02(\mathrm{~s}, 1 \mathrm{H}$, $\mathrm{CH}-4), 6.96$ (s, 2H, $\mathrm{NH}_{2}$ ), 7.46-7.94 (m, 4H, ArH) ppm. Anal. calcd. for $\mathrm{C}_{18} \mathrm{H}_{17} \mathrm{~N}_{3} \mathrm{O}_{4}: \mathrm{C} 63.71, \mathrm{H}$ 5.05, N 12.38; found C 63.80, H 5.11, N $12.43 \%$.

2-Amino-3-cyano-7,7-dimethyl-4-(3-nitrophenyl)-5-oxo-4H-5,6,7,8-tetrahydrobenzopyran (4l). IR (KBr): $v_{\max }=3400,3300,3196,2200,1696,1600 \mathrm{~cm}^{-1} .{ }^{1} \mathrm{H}$ NMR (DMSO-d $)$ ): $\delta_{\mathrm{H}}=1.00\left(\mathrm{~s}, 3 \mathrm{H}, \mathrm{CH}_{3}\right), 1.06\left(\mathrm{~s}, 3 \mathrm{H}, \mathrm{CH}_{3}\right), 2.26(\mathrm{~s}, 2 \mathrm{H}, \mathrm{CH}-8), 2.48-2.52(\mathrm{~m}, 2 \mathrm{H}, \mathrm{CH}-6), 4.54(\mathrm{~s}$, $1 \mathrm{H}, \mathrm{CH}-4), 5.98\left(\mathrm{~s}, 2 \mathrm{H}, \mathrm{NH}_{2}\right), 7.42-7.96(\mathrm{~m}, 4 \mathrm{H}, \mathrm{ArH}) \mathrm{ppm}$. Anal. calcd. for $\mathrm{C}_{18} \mathrm{H}_{17} \mathrm{~N}_{3} \mathrm{O}_{4}: \mathrm{C}$ 63.71, H 5.05, N 12.38; found C 63.80, H 4.98, N $12.43 \%$.

2-Amino-3-cyano-7,7-dimethyl-4-(4-nitrophenyl)-5-oxo-4H-5,6,7,8-tetrahydrobenzopyran (4m). IR (KBr): $v_{\max }=3400,3300,3190,2200,1690,1600 \mathrm{~cm}^{-1} .{ }^{1} \mathrm{H}$ NMR (DMSO-d $)$ : $\delta_{\mathrm{H}}=1.02\left(\mathrm{~s}, 3 \mathrm{H}, \mathrm{CH}_{3}\right), 1.06\left(\mathrm{~s}, 3 \mathrm{H}, \mathrm{CH}_{3}\right), 2.26(\mathrm{~s}, 2 \mathrm{H}, \mathrm{CH}-8), 2.48-2.52(\mathrm{~m}, 2 \mathrm{H}, \mathrm{CH}-6), 4.55(\mathrm{~s}$, $1 \mathrm{H}, \mathrm{CH}-4), 6.04\left(\mathrm{~s}, 2 \mathrm{H}, \mathrm{NH}_{2}\right), 7.46-8.06(\mathrm{~m}, 4 \mathrm{H}, \mathrm{ArH}) \mathrm{ppm}$. Anal. calcd. for $\mathrm{C}_{18} \mathrm{H}_{17} \mathrm{~N}_{3} \mathrm{O}_{4}: \mathrm{C}$ 63.71, H 5.05, N 12.38; found C 63.76, H 5.11, N $12.33 \%$.

2-Amino-3-cyano-7,7-dimethyl-4-(4-methoxyphenyl)-5-oxo-4H-5,6,7,8-tetrahydrobenzo-

pyran (4n). IR (KBr): $v_{\max }=3380,3188,2962,2200,1680,1656,1598 \mathrm{~cm}^{-1} .{ }^{1} \mathrm{H}$ NMR (DMSO$\left.d_{6}\right): \delta_{\mathrm{H}}=0.96\left(\mathrm{~s}, 3 \mathrm{H}, \mathrm{CH}_{3}\right), 1.02\left(\mathrm{~s}, 3 \mathrm{H}, \mathrm{CH}_{3}\right), 2.12(\mathrm{~d}, 1 \mathrm{H}, J=16.0 \mathrm{~Hz}, \mathrm{CH}-8) 2.22(\mathrm{~d}, 1 \mathrm{H}$, $J=16.0 \mathrm{~Hz}, \mathrm{CH}-8), 2.46-2.56$ (m, 2H, CH-6), 3.72 (s, 3H, OCH $), 4.46$ (s, 1H, CH-4), 6.84 (d, 2H, $J=8.0 \mathrm{~Hz}, \mathrm{ArH}), 6.90\left(\mathrm{~s}, 2 \mathrm{H}, \mathrm{NH}_{2}\right), 7.04(\mathrm{~d}, 2 \mathrm{H}, J=8.0 \mathrm{~Hz}, \mathrm{ArH}) \mathrm{ppm}$. Anal. calcd. for $\mathrm{C}_{19} \mathrm{H}_{20} \mathrm{~N}_{2} \mathrm{O}_{3}$ : C 70.35, H 6.21, N 8.64; found C 70.39, H 6.27, N 8.68 \%.

2-Amino-3-cyano-7,7-dimethyl-4-(4-methylphenyl)-5-oxo-4H-5,6,7,8-tetrahydrobenzopyran (4o). IR (KBr): $v_{\max }=3420,3330,2950,2190,1678,1640,1600 \mathrm{~cm}^{-1} .{ }^{1} \mathrm{H}$ NMR (DMSO$\left.d_{6}\right): \delta_{\mathrm{H}}=0.97\left(\mathrm{~s}, 3 \mathrm{H}, \mathrm{CH}_{3}\right), 1.04\left(\mathrm{~s}, 3 \mathrm{H}, \mathrm{CH}_{3}\right), 2.10(\mathrm{~d}, 1 \mathrm{H}, J=16.0 \mathrm{~Hz}, \mathrm{CH}-8) 2.22(\mathrm{~d}, 1 \mathrm{H}$, $J=16.0 \mathrm{~Hz}, \mathrm{CH}-8$ ), 2.26 (s, 3H, $\mathrm{CH}_{3}$ ), 2.46-2.56 (m, 2H, CH-6), 4.32 (s, 1H, CH-4), 6.94 (s, 2H, $\left.\mathrm{NH}_{2}\right), 7.02(\mathrm{~d}, 2 \mathrm{H}, J=8.0 \mathrm{~Hz}, \mathrm{ArH}), 7.08(\mathrm{~d}, 2 \mathrm{H}, J=8.0 \mathrm{~Hz}, \mathrm{ArH}) \mathrm{ppm}$. Anal. calcd. for $\mathrm{C}_{19} \mathrm{H}_{20} \mathrm{~N}_{2} \mathrm{O}_{2}$ : C 74.00, H 6.54, N 9.08; found C 74.10, H 6.57, N 9.13\%.

2-Amino-3-cyano-7,7-dimethyl-4-(3,4-methylenedioxyphenyl)-5-oxo-4H-5,6,7,8-tetrahy-

drobenzopyran (4p). IR (KBr): $v_{\max }=3370,3206,2938,2204,16501607 \mathrm{~cm}^{-1}$. ${ }^{1} \mathrm{H}$ NMR $\left(\mathrm{DMSO}-d_{6}\right): \delta_{\mathrm{H}}=0.96\left(\mathrm{~s}, 3 \mathrm{H}, \mathrm{CH}_{3}\right), 1.02\left(\mathrm{~s}, 3 \mathrm{H}, \mathrm{CH}_{3}\right), 2.12(\mathrm{~d}, 1 \mathrm{H}, J=16.0 \mathrm{~Hz}, \mathrm{CH}-8) 2.24(\mathrm{~d}$, $1 \mathrm{H}, J=16.0 \mathrm{~Hz}, \mathrm{CH}-8), 2.46-2.56$ (m, 2H, CH-6), 4.27 (s, 1H, CH-4), 5.96 (s, 2H, $\left.\mathrm{OCH}_{2} \mathrm{O}\right), 6.92$ $\left(\mathrm{s}, 2 \mathrm{H}, \mathrm{NH}_{2}\right), 7.02-7.16(\mathrm{~m}, 3 \mathrm{H}, \mathrm{ArH})$ ppm. Anal. calcd. for $\mathrm{C}_{20} \mathrm{H}_{18} \mathrm{~N}_{2} \mathrm{O}_{4}: \mathrm{C}$ 67.45, $\mathrm{H} \mathrm{5.36,} \mathrm{N}$ 8.28; found C 67.52, H 5.43, N $8.34 \%$.

\section{Acknowledgements}

This project was supported by the National Natural Science Foundation of China, Educational Ministry of China, Educational Department of Hebei Province, Science and Technology Commission of Hebei Province. 


\section{References}

1. (a) Bonsignore, L.; Loy, G..; Secci, D.; Calignano, A. Eur. J. Med. Chem. 1993, 28, 517. (b) Bloxham, J.; Dell, C. P.; Smith, C. W. Heterocycles 1994, 38, 399. (c) Nawwar, G. A. M.; Abdelrazek, F. M.; Swcllam, R. H. Arch. Pharm. 1991, 342, 875. (d) Zamocka, J.; Misikova, E.; Durinda, J. Pharmazie 1991, 46, 610.

2. Armetso, D.; Horspool, W. M.; Martin, N.; Ramos, A.; Seaone, C. J. Org. Chem. 1989, 54, 3069.

3. Hatakeyama, S.; Ochi, N.; Numata, H.; Takano, S. J. Chem. Soc., Chem. Commun. 1988, 1202.

4. O'Callaghan, C. N.; Marry, T. B. H. J. Chem. Res. Synop. 1995, 214.

5. (a) Kandeel, Z. E.; Farag, A. M.; Shaaban, M. R.; Elnagdi, M. H. Heteroatom Chem. 1996, 7, 35. (b) Al-Ashmawi, M. I.; El-Sadek, M.; Sakr, S. M.; El-Sawah, M. Egypt. J. Pharm. Sci. 1992, 33, 849. (c) Martin-Leon, N.; Quinteiro, M.; Seoane, C.; Soto, J. L. Liebigs Annalen der Chemie 1990, 101. (d) Sharanina, L. G.; Nesterov, V. N.; Klokol, G. V.; Rodinovskaya, L. A.; Shklover, V. E.; Sharanin, Y. A.; Struchkov, Y. T.; Promonenkov, V. K. Zhurnal Organicheskoi Khimii 1986, 22, 1315.

6. (a) Kuthan, J.; Sebek, P.; Bohm, S. Adv. Heterocyclic Chem. 1995, 62, 19. (b) Elnagdi, M. H.; Aal, F. A. M. A.; Yassin, Y. M. J .Prakt . Chem. 1989, 331, 971.

7. (a) Singh, K.; Singh, J.; Singh, H. Tetrahedron 1996, 52, 14273. (b) Hassanien, A. A.; Zahran, M. A.; Gaby, M. S. A.; Ghorab, M. M. J . Indian Chem. Soc. 1999, 76, 350.

8. Wang, X. S.; D. Shi, Q.; Tu, S. J.; Yao, C. S. Synth. Commun. 2003, 33, 119.

9. Tu, S. J.; Jiang, H.; Zhuang, Q. Y.; Miao, C. B.; Shi, D. Q.; Wang, X. S.; Gao, Y. Chin. J. Org. Chem. 2003, 23, 488.

10. (a) Li, C. J. Chem. Rev. 1993, 93, 20. (b) Li, C. J. Chem. Rev. 2005, 105, 3095. (c) Li, C. J.; Chen, L. Chem. Soc. Rev. 2006, 35, 68. (d) Lubineau, A. Chem. Ind. 1996, 123. (e) Meijer, A.; Otto, S.; Engberts, J. B. F. N. J. Org. Chem. 1998, 63, 8989. (f) Ballin, R.; Bosica, G. Tetrahedron Lett. 1996, 37, 8027.

11. Breslow, R.; Maitra, U.; Rideout, D. Tetrahetron Lett. 1983, 24, 1901.

12. (a) Severance, D. L.; Jorgensen, W. L. J. Am. Chem. Soc. 1992, 114, 10966. (b) Brandes, E. B.; Grieco, P. A.; Gajewski, J. J. J. Org. Chem. 1989, 54, 515.

13. (a) Lubineau, A. J. Org. Chem. 1986, 51, 2142. (b) Mori, Y.; Manabe, K.; Kobayashi, S. Angew. Chem. Int. Ed. 2001, 40, 2815.

14. Li, C. J.; Chan, T. H. Organic Reaction in Aqueous Media, Wiley: New York, 1997.

15. Kool, E. T.; Breslow, R. J. Am. Chem. Soc. 1988, 110, 1596.

16. (a) Armando, C.; Carlos, F. B. Tetrahedron Lett. 2003, 44, 1923. (b) Yi, L.; Zou, J.; Lei, H.; Lin, X.; Zhang, M. Org. Prep. Proc. Int. 1991, 23, 673. (c) Manabe, K.; Mori, Y.; Kobayashi, S. Tetrahedron 2001, 57, 2537.

17. Silva, F. M.; Jones, J. J. J. Braz. Chem. Soc. 2001, 12, 135.

18. (a) Wang, X. S.; Zhang, M. M.; Zeng, Z. S.; Shi, D. Q.; Tu, S. J.; Wei, X. Y.; Zong, Z. M. 
Arkivoc 2006, 7(ii), 117. (b) Jin, T. S.; Zhao, R. Q.; Li, T. S. Arkivoc 2006, 7(xi), 176. (c) Stefani, H. A.; Pereira, C. M. P.; Dorr, F. A.; Cell, R. Arkivoc 2005, 6(ii), 19. (d) Jin, T. S.; Zhang, J. S.; Xiao, J. C.; Wang, A. Q.; Li, T. S. Synlett 2004, 866. (e) Jin, T. S.; Wang, A. Q.; Cheng, Z. L.; Li, T. S. Synth. Commun. 2005, 35, 2339. (f) Jin, T. S.; Zhang, J. S.; Guo, T. T.; Wang, A. Q.; Li, T. S. Synthesis 2004, 2001. (g) Jin, T. S.; Wang, A. Q.; Cheng, Z. L.; Zhang, J. S.; Li, T. S. Synth. Commun. 2005, 35, 137. (h) Naik, S.; Bharttacharjya, G.; Kavala, V. R.; Patel, B. K. Arkivoc 2004, 5(i), 55.

19. (a) Shi, D. Q.; Mou, J.; Zhuang, Q. Y.; Wang, X. S. J. Chem. Res. 2004, 821. (b) Sharanina, L. G.; Nesterov, V. N.; Klokol, G. V.; Rodinovskaya, L. A.; Shklover, V. E.; Sharanin, Y. T.; Struchkov, Y. T.; Promoonenkov, V. E. Zh.Org. Khim. 1986, 22, 1315. (c) Jin, T. S.; Wang, A. Q.; Wang, X.; Zhang, J. S.; Li. T. S. Synlett 2004, 871. 